\title{
Machine Vision Based Information Collection of Mobile Manipulator for Moving Object Grasping
}

\author{
CHEN Naijian, SONG Fangzheng, LI Guoping, ZHAO Honghua \\ School of Mechanical Engineering \\ University of Jinan \\ Jinan 250022, Shandong, P.R. China \\ e-mail: chnjian@126.com
}

\begin{abstract}
To solve the problem of reliability and real time in visual perception of the mobile manipulator, a visual based information collection system integrated edge detection algorithm and tracking algorithm is proposed. In the vision system, an adaptable threshold edge detection algorithm based on the small world network is presented to improve the reliability and accuracy of edge detection and image segmentation, and the Meanshift algorithm is used to track the mobile objective in real time. Firstly, the edge detection algorithm based on small world phenomenon can adapt the threshold from the global to the local and it improves the ability of the mobile manipulator to find the moving object. Secondly, the edge detection results are used to initialize the target area of Meanshift algorithm, which then tracks the moving objective and extracts parameters' information automatically and real time. The proposed vision system not only meets the need of a mobile manipulator operating on moving objectives but also provides an effective method to find and track objectives automatically.
\end{abstract}

Keywords-mobile manipulator; moving object; visual tracking; visual detecting

\section{INTRODUCTION}

Due to own the high mobility combined with dexterous manipulation abilities, the mobile manipulators have attracted considerable attention in the area of industrial, agriculture, public service communities and so on [1-6]. There are some assigned targets to operate replacing human in various tedious, repetitive and hazardous task, and cameras are also selected to detect targets. As targets are divided into the moving object and the static one according to different movement patterns. Most of the researches on the mobile manipulator operating targets are developed on static objects, and there are very few researches on moving objects. Han, etc. [7] developed a differential driving mobile robot to capture a moving object. However, they considered only tracking errors of the mobile robot. Yamamoto and Yun [8] developed a mobile manipulator performing the task of pushing against and following a moving surface in environments. Dissimilar to other research, we pay more attention to the mobile manipulator operating on the moving object. The mobile manipulator is designed to perform the task of tracking and operating against the moving object in environments such as an automatic fire searching and suppression system for large spaces or the robotic system for the fire fighting in tunnels. The key research issue in the mobile manipulator operating on a moving object is the moving object identifying and tracking visually. The vision based information collection system of the mobile manipulator for moving object grasping is developed to find and locate the moving object, measure the distance between the end-effector and the moving object, perceive the speed of the moving object and its' moving direction, and it would help to make the curved motion planning and capture the target subjected to nonholonomic constricts.

\section{SYSTEM WORKING PRINCIPLE}

A mobile manipulator $[9,10]$ in this study is a redundant 2-DOF manipulator mounted on a wheeled mobile platform, its schematic picture is shown in Figure 1.

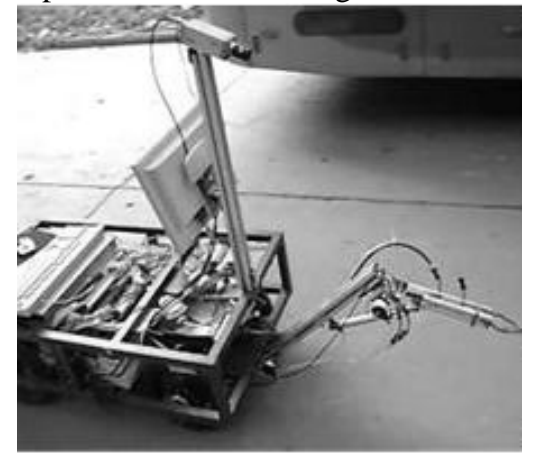

Figure 1. Schematic picture of the mobile manipulator

The mobile manipulator online detects the moving object grasping in the working space by the camera system. Once the moving object is found, the visual system can identify such information as location, velocity of the target and send that information to the motion planning. Then the mobile manipulator is equipped with an image acquisition and processing system, from which the mobile manipulator can find the moving object and measure the distance, the speed and the direction of the moving object. The detection and tracking system is composed of a video camera typed Panasonic NASA NSJ-CC382 with AVENIR SSV0358 lens and an external frame grabber box MV-U2000. The working process of the mobile manipulator is described by Figure 2 . 


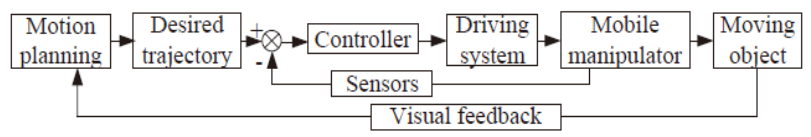

Figure 2. Working principle of the mobile manipulator

\section{VISION BASED INFORMATION COLLECTION STSTEM}

The moving object detection and tracking system based on the machine vision is an essential element to the moving object grasping. The main purpose of the detection and tracking system is to identify and track the moving target, collect information about the object and contribute to make a reasonable motion planning and motion control.

Figure 3 depicts the schematic diagram of the vision based moving object detection and tracking system of the mobile manipulator.

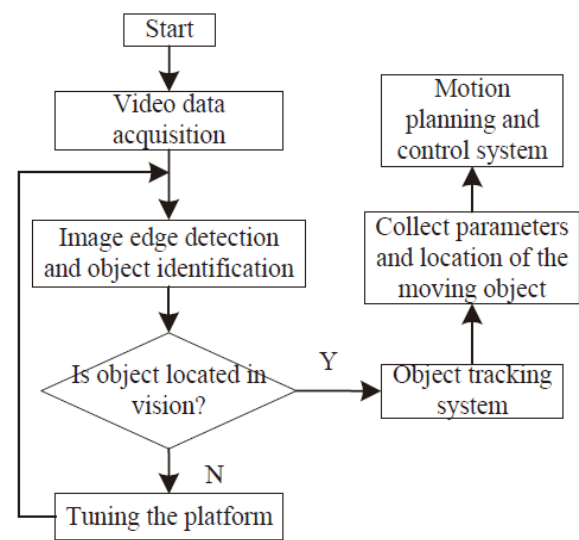

Figure 3. The schematic diagram of the moving object detection and tracking system

The vision system comprises of an image detection algorithm and moving target tracking system. The edge detection algorithm is selected to identify the profile of the target against the background, and to complete the image segmentation. If the moving object is found in image series, image segmentation results are used to initialize the target area in the first frame of the tracking algorithm. Otherwise, the mobile platform needs to be turned around to find the moving target. According to changes in location and size of the target from one frame to the next, the motion planning system and control system can collect the parameters about the target. It is facilitated for the mobile manipulator to operate on the object.

The image edge detection and segmentation algorithm based on small world phenomenon [11] and Meanshift tracking algorithm[12,13] are integrated into the vision based moving object detection and tracking system of the NMM. The edge detection algorithm generalizes smallworld phenomenon to image edge detection and segmentation. It begins by computing the optimal threshold, based on global image features. Through pre-setting the probability of clustering, the algorithm could change the threshold in a certain range and apply it from overall features to partial attributes, and segment the image from rough to detail. Hence, it can improve the ability of the edge detection and segmentation of the vision system in different environments. The results of image segmentation are adopted to initialize the first frame of the Meanshift algorithm. Thus, the integrated vision system has the advantages in autonomy and the real time of the object detection and tracking.

Generally speaking, the moving object can be measured manually. As the moving object is assigned in size, the ratio of the object to its image is nearly proportional to its distance from the camera, and the tracking system can be calibrated to measure the distance according to images of the object. Figure 4 describes measured distances in the same and offset directions.

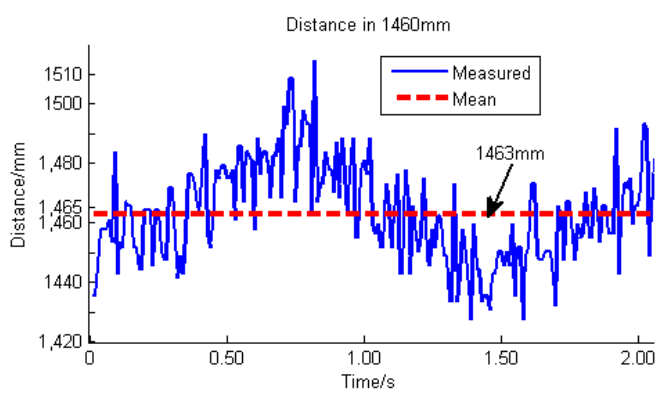

(a) Same direction

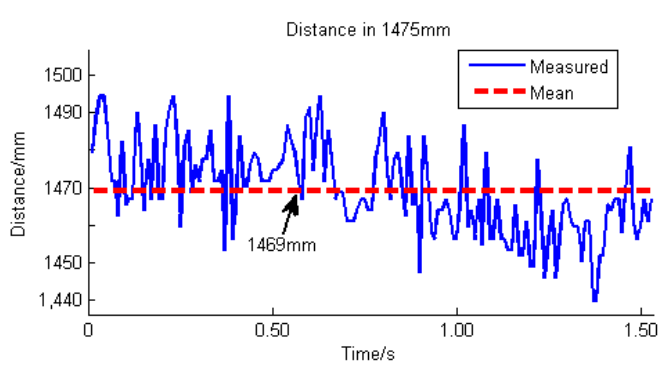

(b) Offset direction

Figure 4. Measured distance in different directions.

Figure 4 a) shows the measured and mean distances between the mobile manipulator and the target compared with the real distance $1460 \mathrm{~mm}$ in the same direction. The measured distance is fluctuated in $1430 \sim 1510 \mathrm{~mm}$, and its mean distance is $1463 \mathrm{~mm}$ near to the real distance $1460 \mathrm{~mm}$. Figure 4 b) shows the measured and mean distances between the mobile manipulator and the target compared with the real distance $1475 \mathrm{~mm}$ in the offset direction. The measured distance is fluctuated in 1440 1495 mm, and its mean distance is $1469 \mathrm{~mm}$ near to the real distance $1475 \mathrm{~mm}$. Accordingly, the mean measured distance can be taken as the real distance sends to the motion planning system. To calibrate the ratio of the object to images, the relation between distance and pixels is described in Figure 5. 


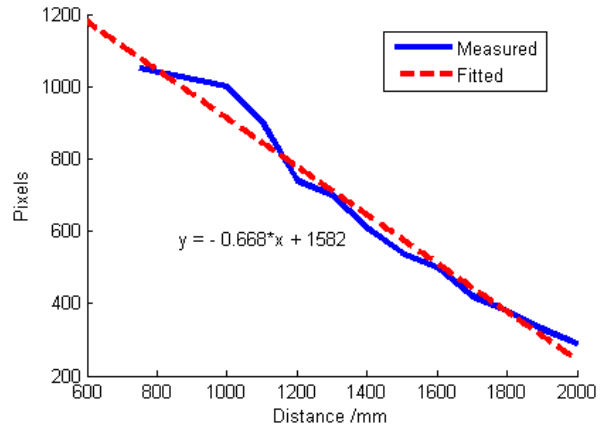

Figure 5. Relation between distance and pixels.

Figure 5 describes the ratio of the object to its image is nearly proportional to its distance from the camera. The relationship between distance and pixels can be taken as:

$$
y=-0.668 x+1582
$$

Where, $x$ denotes the distance, $y$ is pixels in the images. Then, the distance can be measured as the system tracks the target autonomously.

\section{EXPERIMENTS}

The experiments were performed to show the effectiveness of the vision based information collecting system to find and detect the moving object on the road outside. In experiments, the camera is constructed on the front of the mobile manipulator, the mobile manipulator is about $2 \mathrm{~m} / \mathrm{s}$ in velocity and the moving object is set up to $1 \mathrm{~m} / \mathrm{s}$ in velocity correspondingly.

Figure 6 is the interface of the vision based information system in the mobile manipulator and Figure 7 describes the experimental results of the information collecting system tracking the moving object outdoors.

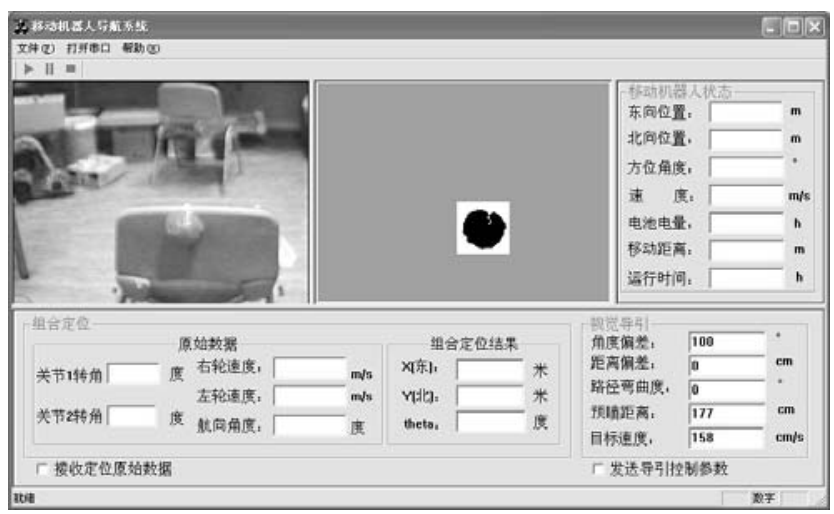

Figure 6. Interface of visual system in the mobile manipulator
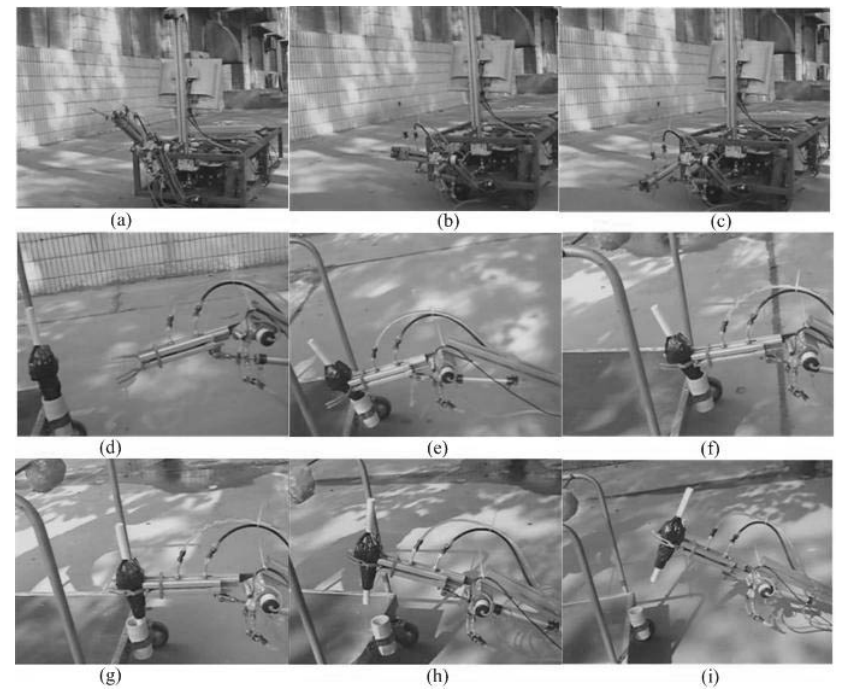

Figure 7 Experimental results

Figure 7 shows the information collecting system could find the moving object in time, calculate the distance between the object and the mobile manipulator, which help the mobile manipulator to determine the preferred operation region and parameters, and the mobile manipulator finished to capture the moving object at the right time eventually.

\section{CONCLUSION}

In this paper, the information collection system of the mobile manipulator for moving object grasping has been discussed. Using the adaptable threshold edge detection algorithm based on small world phenomenon and Meanshift algorithm, the proposed information collection system can find and tracking the moving object automatically. Experimental results show that the proposed vision system improves the ability of the mobile manipulator to find moving objects and extracts parameters' information automatically and real time. The proposed vision system not only meets the need of the mobile manipulator operating on moving objectives but also provides an effective method to find and track objectives, and the vision system has a high engineering value.

\section{ACKNOWLEDGMENT}

The present work is supported by the Youth Scientist Incentive Foundation of Shandong Province (No.BS2011DX003), the Doctor Foundation of University of Jinan (No.XBS1101) and Research Development Fund of University of Jinan (No.XKY1203).

\section{REFERENCES}

[1] Sakai, S. , Iida, M., Osuka, K. and Umeda, M. "Design and control of a heavy material handling manipulator for agricultural robots," Autonomous Robots 2008, 25(3), 189-204.

[2] Datta, S. and Ray, R. and Banerji, D., "Development of autonomous mobile robot with manipulator for manufacturing environment," International Journal of Advanced Manufacturing Technology 2008, 38(5-6), 536-542. 
[3] Hamner, B., Koterba, S.,et al., "An autonomous mobile manipulator for assembly tasks," Autonomous Robots 2010,28(1), 131-149.

[4] Chung, W.J., G. Kim, and M. Kim, "Development of the multifunctional indoor service robot PSR systems," Autonomous Robots 2007, 22(1), 1-17.

[5] Deegan, P., Grupen, R., Hansonet, A., al., "Mobile manipulators for assisted living in residential settings," Autonomous Robots 2008,24(2),

[6] Chen, N. J. , Wang, S. A., Zhang, J. H. "Note: Development of a compact electromagnetic hydraulic pump for a microrobot joint driving system. ” Review of Scientific Instruments : 81,2010,046105.

[7] Han, S. and Choi, B. and Lee, J. "A precise curved motion planning for a differential driving mobile robot," Mechatronics 2008, 18(9), 486-494.

[8] Yamamoto, Y. and Yun, X. "Control of mobile manipulators following a moving surface," Proc. Conf. IEEE Int Robotics and Automation,Atlanta, GA, USA, May 1993, 2-6.
[9] Naijian Chen,Guoping Li, Fangzhen Song, Xuan Sun and Changsheng Ai."An immune small world algorithm for motion planning of the mobile manipulator," International Journal of Robotics and Automation, in press.

[10] Naijian Chen, Fangzhen Song, Guoping Li, Xuan Sun and Changsheng Ai." An Adaptive Sliding Mode Backstepping Control for the Mobile Manipulator with Nonholonomic Constraints," unpublished.

[11] Chen, Naijian,Wang, Sun'an."An image edge detection and segmentation algorithm based on small world phenomenon," 2008 3rd IEEE Conference on Industrial Electronics and Applications, Singapore, 2008:2272-2277.

[12] Song, D., Zhao, B.J., and Tang, L.B.: "Mean-Shift algorithm fused with corner feature and color feature for target tracking," Systems Engineering and Electronics, 2012, 34, (1), pp. 199-203

[13] Chouhan, M.K., Mishra, R., and Nitnawwre, D.: "Multiple Target Tracking with the help of Mean Shift Algorithm," International Journal of Advanced Research in Computer Engineering \& Technology (IJARCET), 2012, 1, (4), pp. pp: 649-652 